\title{
Personalized Medicine
}

\section{Importance of race and ethnicity in individuals' use of and responses to genomic information}

\author{
"Both individual- and system-level factors likely contribute to \\ differences by race and ethnicity in use of and responses to genomic \\ information."
}

\begin{abstract}
First draft submitted: 14 September 2015; Accepted for publication: 28 September 2015; Published online: 14 December 2015
\end{abstract}

Keywords: access $\bullet$ ethnicity $\bullet$ genetic testing $\bullet$ genomic information $\bullet$ health disparities - race

Genomic information is becoming increasingly important in healthcare with possible uses including the personalization of prevention and treatment recommendations and motivation for health behavior change [1]. In order to translate genomic information effectively to clinical and public health contexts, we need to understand how individuals from different population subgroups use this information as costly or complicated advances may in fact exacerbate health disparities. Thus, a critical issue as the integration of genomic information expands will be to investigate use of and responses to this information across racial and ethnic groups to avoid widening existing disparities in access to and uptake of health information and services that impact health outcomes [1].

In a previous study, we found evidence that race and ethnicity affect responses to information about genomic risk for common, chronic conditions in a medically underserved patient population. Participants viewed a hypothetical vignette that presented risk for either heart disease or diabetes following a genomic assessment. We found that nonHispanic black participants had significantly greater interest in discussing the genomic risk information with a doctor compared with white participants, and both non-Hispanic black and Hispanic participants had greater interest in discussing the information with family than white participants. In addition to these communication responses, black and Hispanic participants reported that they intended to change fewer health behaviors than white participants based on the information, although Hispanic participants were more interested in receiving a genomic assessment than white participants [2]. These findings therefore suggested that both interest in accessing genomic information and resulting behavioral responses may differ by race and ethnicity. Although the research base is limited, other studies have also examined how race and ethnicity might affect responses to genomic information. For example, a study investigating use of risk reduction strategies following genetic counseling and testing for $B R C A 1$ among female members of an African-American kindred showed that surveillance was preferred to prophylactic surgery and chemoprevention for cancer risk reduction. The findings also revealed suboptimal provider-patient communication in this patient group [3]. The evidence in this area suggests that responses to genomic information may vary by race and ethnicity, and that factors such as provider-patient communication about the information may contribute to these differences.

Related work has suggested that individuals from minority racial and ethnic groups may be less likely to access and use genetic testing and, therefore, genomic information. For example, a number of studies have shown lower use of genetic testing among individuals from racial and ethnic minority groups

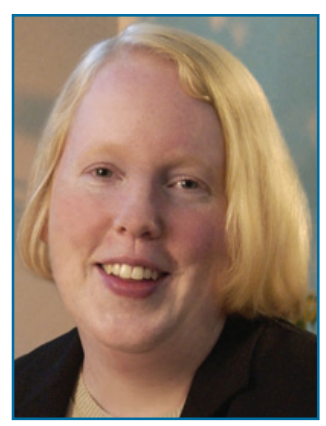

Kimberly A Kaphingst Author for correspondence: Huntsman Cancer Institute, University of Utah, Salt Lake City, UT 84112, USA and

Department of Communication, University of Utah, Salt Lake City, UT 84112, USA

Tel.: +1 8012135724

kim.kaphingst@hci.utah.edu

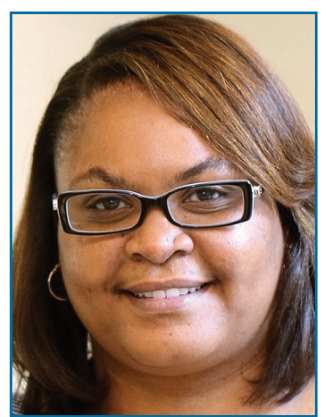

Melody S Goodman Division of Public Health Sciences, Washington University School of Medicine, St Louis, MO 63110, USA 
compared with whites [4]. Interestingly, disparities in use remain even when barriers of ascertainment and cost are minimized, suggesting that both access and decisions to use genetic services may differ by race and ethnicity. Among a population-based sample of healthy adults aged $25-40$ years with access to healthcare in the USA, African-American individuals were less likely to opt to receive a free multiplex genetic susceptibility test [5]. A recent randomized trial comparing mode of delivery of genetic counseling (in-person vs telephone-based) for hereditary breast and ovarian cancer at multiple USA sites found that the effect of mode of delivery differed by race; minority women assigned to telephone counseling were the least likely to complete genetic testing [6].

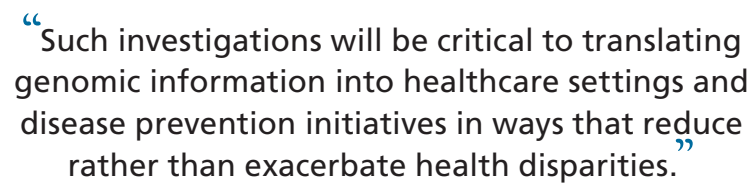

A number of possible mechanisms underlying racial and ethnic differences in access to and use of genomic information and responses to this information have been advanced, including both individual- and systemlevel factors. Individual-level factors identified include awareness, knowledge, attitudes and beliefs. A number of prior studies have suggested that individuals from minority racial and ethnic groups have lower knowledge about and more negative attitudes related to genetic testing, which could affect both use of genetic services and responses to the information $[7,8]$. However, some contradictory evidence has been found. A national telephone survey of US adults showed that although there were some differences in genetic knowledge by racial and ethnic group, many misunderstandings about genetic concepts were shared across groups [9]. Negative attitudes may be due in part to lack of awareness or knowledge. In a focus group study with Latinos, awareness of familial breast and ovarian cancer and availability of genetic services were low, but after participants received information they held favorable attitudes toward genetic services [10]. Culture is likely to play a critical role in shaping individuals' awareness, beliefs and attitudes about genomic information. Cultural differences in fatalism, temporal orientation, spirituality and beliefs about illness and prevention might affect differences in use of and responses to genomic information across racial and ethnic groups [11]. Acculturation has also been shown to affect awareness of and attitudes toward genetic testing among Latinos [7]. Disentangling how these various individual level factors affect each other, and how culture influences these factors, will be critical to understanding differences in downstream outcomes by race and ethnicity.
Although more research has investigated how individual-level factors might affect use of and responses to genomic information, a few studies have pointed to the importance of system-level factors. In an investigation of providers' perceptions of genetic counseling and testing for BRCA1/2 in African-American women, providers identified both individual- and system-level barriers to use of genetic services [12]. Healthcare system barriers such as insurance barriers might impact not only access to genetic services but also responses to genomic information if access to healthcare providers is impaired. Lower levels of trust in the healthcare system among individuals from minority racial and ethnic groups may lead to greater concerns about misuse of genetic information and racial discrimination based on genetic testing $[8,11]$.

Both individual- and system-level factors likely contribute to differences by race and ethnicity in use of and responses to genomic information. A recent crosssectional survey of women diagnosed with nonmetastatic breast cancer showed a strong desire for genetic testing among Latinas, but also highlighted that minority patients were significantly more likely to have an unmet need for discussion of genetic testing with providers [13]. Others have called for empirical research on disparities in genetic testing that moves beyond descriptive studies to differentiate between disparities in access to genetic services and differences in use of those services that are due to individual preferences and cultural values (first-generation health disparities research) [14,15]. We would add the importance of multilevel studies examining how individual- and systemlevel factors interact to affect both use of and responses to genomic information (second generation). In addition, we need to move toward higher generations of health disparities research in the area of genomics to examine the effectiveness of interventions aimed to reduce disparities (third generation) and address health equity using community-based participatory research approaches (fourth generation) [16,17].

A number of additional areas of research are needed to better understand how race and ethnicity affect use of and responses to genomic information. Our understanding of the role of race and ethnicity has been hampered by the under representation of individuals from minority groups in genetic research [5], and we need to develop outreach approaches that are culturally appropriate for individuals and communities from different population subgroups. More work is particularly needed to understand use of and responses to different types of genomic information among population subgroups such as Pacific Islanders and Native communities. In addition, exploration of the heterogeneity within racial and ethnic groups is critical. One such 
study has indicated important areas of heterogeneity within Hispanic subethnicities regarding preferences for information about genetic testing [18], but more research is needed to build upon this. Heterogeneity within racial and ethnic group according to factors such as health literacy, numeracy, socioeconomic status, country of origin and geographic region are also critical to examine. Finally, much of the prior work in this area has focused on genetic testing for hereditary cancers, and investigations are needed with regard to newer technologies with powerful implications for personalized medicine, such as multigene panels, genome sequencing and pharmacogenomics.

Addressing the research gaps outlined above is critical to the development of effective communication approaches to increase appropriate use of genetic services and translate research in genomics into improvements in clinical care and public health [1]. Research to develop and test culturally appropriate communication approaches for genomic information has shown promise. In a randomized trial comparing culturally tailored genetic counseling and standard genetic counseling among African-American women at moderate or high risk for $B R C A$ mutations, those who received the culturally tailored approach had significantly decreased worry [19]. Understanding how differences in attitudes toward and knowledge of genomic information vary across racial and ethnic groups can also assist in developing strategies to support individuals in

\section{References}

1 McBride CM, Bowen D, Brody LC et al. Future health applications of genomics: priorities for communication, behavioral, and social sciences research. Am. J. Prev. Med. 38(5), 566-561 (2010).

2 Kaphingst KA, Stafford JD, McGowan LDA, Seo J, Lachance CR, Goodman MS. Effects of racial and ethnic group and health literacy on responses to genomic risk information in a medically underserved population. Health Psychol. 34(2), 101-110 (2015).

3 Kinney AY, Simonsen SE, Baty BJ et al. Risk reduction behaviors and provider communication following genetic counseling and BRCA1 mutation testing in an African American kindred. J. Genet. Couns. 15(4), 293-305 (2006).

4 Hall M, Olopade OI. Confronting genetic testing disparities: knowledge is power. J. Am. Med. Assoc. 293, 1783-1785 (2005).

5 Alford SH, McBride CM, Reid RJ, Larson EB, Baxevanis AD, Brody LC. Participation in genetic testing research varies by social group. Public Health Genomics 14, 85-93 (2011).

6 Butrick $M$ et al. Disparities in uptake of BRCA1/2 genetic testing in a randomized trial of telephone counseling. Genet. Med. 17(6), 467-475 (2015).

7 Pagan JA, Su D, Li L, Armstrong K, Asch DA. Racial and ethnic disparities in awareness of genetic testing for cancer risk. Am. J. Prev. Med. 37(6), 524-530 (2009). making informed decisions about the use of genomic information. Intervention research can investigate how effective approaches to assist individuals to understand and act upon genomic information might differ across racial and ethnic groups [20].

In sum, prior research has shown differences by race and ethnicity in use of and responses to genomic information. However, many gaps remain and interdisciplinary research is needed to fully understand these differences and the mechanisms that underlie them. Such investigations will be critical to translating genomic information into healthcare settings and disease prevention initiatives in ways that reduce rather than exacerbate health disparities.

\section{Acknowledgements}

The authors thank $G$ Smith for research assistance.

\section{Financial \& competing interests disclosure}

KA Kaphingst was supported by funding from the Huntsman Cancer Institute and University of Utah and MS Goodman by the Siteman Cancer Center, Barnes-Jewish Hospital Foundation and the Washington University School of Medicine. The authors have no other relevant affiliations or financial involvement with any organization or entity with a financial interest in or financial conflict with the subject matter or materials discussed in the manuscript apart from those disclosed.

No writing assistance was utilized in the production of this manuscript.

8 Singer E, Antonucci T, Van Hoewyk J. Racial and ethnic variations in knowledge and attitudes about genetic testing. Genet. Test. 8(1), 31-43 (2004).

9 Christensen KD, Jayaratne TE, Roberts JS, Kardia SLR, Petty EM. Understandings of basic genetics in the United States: results from a national survey of black and white men and women. Public Health Genomics 13, 467-476 (2010).

10 Kinney AY, Gammon A, Coxworth J, Simonsen SE, ArceLaretta M. Exploring attitudes, beliefs, and communication preferences of Latino community members regarding BRCA1/2 mutation testing and preventive strategies. Genet. Med. 12(2), 105-115 (2010).

11 Peters N, Rose A, Armstrong K. The association between race and attitudes about predictive genetic testing. Cancer Epidemiol. Biomarkers Prev. 13, 361-365 (2004).

12 Graves KD, Christopher J, Harrison TM, Peshkin BN, Isaacs C, Sheppard VB. Providers' perceptions and practices regarding $B R C A 1 / 2$ genetic counseling and testing in African American women. J. Genet. Couns. 20, 674-689 (2011).

13 Jagsi $\mathrm{R}$ et al. Concerns about cancer risk and experiences with genetic testing in a diverse population of patients with breast cancer. J. Clin. Oncol. 33(14), 1584-1591 (2015).

14 Halbert $\mathrm{CH}$ et al. Low rates of African American participation in genetic counseling and testing for BRCA1/2 
mutations: racial disparities or just a difference? J. Genet. Couns. 21(5), 676-683 (2012).

15 Thomas SB, Quinn SC, Butler J, Fryer CS, Garza MA. Toward a fourth generation of disparities research to achieve health equity. Annu. Rev. Public Health. 32(c), 399-416 (2011).

16 Gehlert S, Coleman R. Using community-based participatory research to ameliorate cancer disparities. Health Soc. Work. 35(4), 281-289 (2010).

17 Ford CL, Airhihenbuwa CO. Critical race theory, race equity, and public health: toward antiracism praxis. Am. J. Public Health. 100(3), 693-698 (2010).
18 Vadaparampil ST, McIntyre J, Quinn GP. Awareness, perceptions, and provider recommendation related to genetic testing for hereditary breast cancer risk among at-risk

Hispanic women: similarities and variations by sub-ethnicity. J. Genet. Couns. 19(6), 618-629 (2010).

19 Charles S, Kessler L, Stopfer JE, Domchek S, Halbert CH. Satisfaction with genetic counseling for BRCA1 and BRCA2 mutations among African American women. Patient Educ. Couns. 63, 196-204 (2006).

20 Kaphingst KA, Lachance CR, Gepp A, D’Anna LH, RiosEllis B. Educating underserved Latino communities about family health history using lay health advisors. Public Health Genomics 14(4-5), 211-221 (2011). 